Revista Brasileira de Agricultura Irrigada v.9, nº.1, p. 1 - 13, 2015

ISSN 1982-7679 (On-line)

Fortaleza, CE, INOVAGRI - http://www.inovagri.org.br

DOI: $10.7127 /$ rbai.v9n100268

Protocolo 268.14 - 20/10/2014 Aprovado em 15/12/2014

\title{
DESEMPENHO DO SISTEMA DE ASSESSORAMENTO AO IRRIGANTE - S@ I PARA A GESTÃO DA ÁGUA EM ÁREAS IRRIGADAS: BENEFÍCIOS AOS IRRIGANTES E AO DISTRITO
}

Sílvio Carlos Ribeiro Vieira Lima ${ }^{1}$, Francisco de Souza $^{2}$, José Antônio Frizzone ${ }^{3}$, Débora Costa Camargo ${ }^{4}$, José Aguiar Beltrão Júnior ${ }^{5}$, Ana Kelliane Silva do Nascimento ${ }^{6}$

\section{RESUMO}

O Serviço de Assessoramento ao Irrigante - SAI é um projeto que tem o objetivo de transmitir informações aos irrigantes sobre o manejo correto da irrigação. Para realizar o manejo da irrigação do SAI, foi desenvolvido em 2011 um software específico para o sistema. O sistema de computação criado é o Sistema Assessoramento ao Irrigante - S @ I. Este sistema realiza os cálculos necessários para o envio da informação diária de quanto irrigar e possibilita uma rede social entre a gerência do distrito e os irrigantes. Para validar as ferramentas do Sistema este trabalho objetivou avaliar a adoção do S @I e também apresentar indicadores econômicos como a Produtividade de Água (WP). Em relação aos lotes, pode-se concluir, de acordo com as entrevistas, que a utilização do Sistema S@I para o envio das informações aos irrigantes gerou visualmente benefícios aos produtores. Pelas análises realizadas, a WP variou de $0,68 \mathrm{R} \$ . \mathrm{m}^{-3}$ a $1,15 \mathrm{R} \$ . \mathrm{m}^{-3}$ para o DIBAU e de $1,19 \mathrm{R} \$ . \mathrm{m}^{-3}$ a 1,38 $\mathrm{R} \$ . \mathrm{m}^{-3}$ quando simulado pelo S@I. Em todas as situações a WP S@I foi mais alta do que a WP DIBAU demonstrando que haverá maior valor de produção por metro cúbico de água se os produtores seguirem as recomendações do Sistema S@I. Desta forma, mesmo com a falta de dados para uma melhor conclusão em relação aos lotes de cada irrigante, somente com a avaliação da WP, o Sistema S@I se mostrou eficiente a nível global, comprovado pelo benefício que este Sistema pode fornecer ao Distrito na gestão da água levando em consideração o demandado pelas culturas implantadas.

Palavras-chave: tecnologia de informação, produtividade de água, indicadores de desempenho.

\footnotetext{
${ }^{1}$ Pesquisador do Instituto INOVAGRI, silviocarlos@inovagri.org.br

${ }^{2}$ Eng. Agrônomo, Ph.D, Pesquisador do Instituto INOVAGRI, Fortaleza, CE, fsouza@ufc.br

${ }^{3}$ Professor Titular, ESALQ/USP, frizzone@usp.br

${ }^{4}$ Pesquisadora do Instituto INOVAGRI, debora@inovagri.org.br

${ }^{5}$ Engenheiro Agrônomo, Pesquisador do Instituto INOVAGRI, aguiar@inovagri.org.br

${ }^{6}$ Doutoranda Universidad de Castilla-La Mancha, kelliane@inovagri.org.br
} 


\title{
PERFORMANCE OF THE ADVISORY SYSTEM FOR IRRIGATORS - S@I FOR WATER MANAGEMENT IN IRRIGATED ÁREAS: BENEFITS FOR FARMERS AND TO THE DISTRICT
}

\begin{abstract}
The Advisory Service to the irrigator - SAI is a project that aims to transmit information to the irrigators about the proper irrigation management. To perform the irrigation management of the SAI, a specific software for the system was developed in 2011. The computing system created is the Advisory System for Irrigators - S@I. This system performs calculations required for sending the daily information as "how much" to irrigate and provides a social network between the farmers and the district management. To validate the tools System, this work aimed to evaluate the adoption of S@I and present economic indicators such as Water Productivity (WP). Relating to the lots, it is possible conclude, according to the interviews, that the use of S@I system to send information to the irrigators, visually generated benefits to the producers. By the performed analyzes, the WP ranged from $\mathrm{R} \$ 0.68 \mathrm{~m}^{-3}$ at $\mathrm{R} \$ 1.15 . \mathrm{m}^{-3}$ for DIBAU and R\$1.19 $\mathrm{m}^{-3}, \mathrm{R} \$ 1.38 . \mathrm{m}^{-3}$ when simulated by S@I. In all situations the S@I WP was higher than the DIBAU WP demonstrating that will show higher production value per cubic meter of water if the District and producers follow the recommendations of the S@I System. However, even with the lack of data for a better conclusion for the lots of each irrigator, only with the evaluation of the WP, the S@I system proved a globally effectiveness and the benefit that this system can provide to the District in water management considering the defendant by the implanted crops.
\end{abstract}

Keywords: information technology, water productivity, performance indicators.

\section{INTRODUÇÃO}

O Serviço de Assessoramento ao Irrigante (SAI) é uma ferramenta de apoio importante para as atividades de melhoria e modernização de sistemas de irrigação e de grande utilidade para otimizar o uso da água, da energia e de outros fatores de produção. Com este sistema é possível responder às demandas tecnológicas dos irrigantes e assessorá-los sobre o manejo da irrigação em função da tecnologia existente, do sistema de irrigação utilizado, do estado de cultivo e dos solos, bem como apoiar a melhoria na gestão técnico-econômica das associações de irrigantes para favorecer ao uso eficiente dos recursos, principalmente a água. O SAI pode contribuir através de uma estreita colaboração com os produtores em temas relacionados com o manejo do cultivo, o cálculo de necessidades hídricas, o estabelecimento de calendário de irrigação e as avaliações dos sistemas de irrigação, entre outros aspectos.

Estes serviços têm sua origem na Califórnia, com a criação do primeiro serviço de assessoramento. Esta iniciativa surgiu como uma ferramenta para realizar um uso eficiente da água, constituindo-se no California Irrigation Management Information System (SNYDER, 1984). Outros Serviços destacados são os desenvolvidos no Reino Unido, Itália e Espanha (ORTEGA et al., 2005).

A avaliação de desempenho da irrigação (em nível de agricultor ou de área irrigada) e a análise dos padrões de variação na produção e no uso da água (LOZANO \& MATEOS, 2008) modernizaram as abordagens de pesquisa do SAI. Técnicas como "benchmarking" (termo em inglês de identificação de pontos de referência para a produtividade e eficiência) para os agricultores que são menos produtivos 

GESTÃO DA ÁGUA EM ÁREAS IRRIGADAS: BENEFÍCIOS AOS IRRIGANTES E AO DISTRITO

conferiram eficiência ao SAI (GONZÁLEZDUGO \& MATEOS, 2008; RODRIGUEZDÍAZ et al., 2008). Programas de monitoramento ambiental podem ser incorporados no SAI, bem como os sistemas de vigilância e de avaliação/aprendizagem de práticas de conservação e gestão integrada da água e do solo (ISIDORO et al., 2004; BARROS et al., 2011). De acordo com Lima et al. (2013a), apesar dos esforços significativos realizados em países que são referências no desenvolvimento de projetos de serviços de assessoramento ao irrigante (como regiões dos Estados Unidos, da Espanha e da Austrália) existem várias limitações a serem superadas na implementação de um SAI.

$\mathrm{O}$ Instituto INOVAGRI e o Instituto Nacional de Ciência e Tecnologia em Engenharia da Irrigação (INCT-EI), com sede na ESALQ/USP, realizam desde 2011, um projeto com o objetivo de desenvolver um modelo de Serviço de Assessoramento ao Irrigante - SAI. A área Piloto que serviu de observação e experimentação do Serviço de Assessoramento ao Irrigante - SAI, foi implantada no Distrito de Irrigação do Baixo Acaraú (DIBAU), no estado do Ceará, Brasil. As parcelas produtivas desse Distrito são cultivadas com fruteiras e olerícolas, destinadas ao mercado nacional e à exportação (SANTOS NETO et al., 2011; LIRA et al., 2011; LIMA et al., 2012; LIMA et al., 2013b).

Em relação às atividades necessárias para o alcance dos objetivos deste Projeto é possível citar: 1) Desenvolver um modelo de Serviço de Assessoramento ao Irrigante- SAI para implantação em áreas irrigadas brasileiras; 2) Realizar avaliação de sistemas de irrigação e orientar a maneira correta de se irrigar; 3) Calcular o Tempo de Irrigação para cada irrigante e informar quando e quanto irrigar aos agricultores via SMS, e-mail e WebService.

Dentre os perímetros irrigados que estão localizados no estado do Ceará, o Distrito de
Irrigação do Baixo Acaraú - DIBAU é o segundo maior em termos de demanda hídrica. Apesar da elevada demanda de água, o DIBAU não possuía nenhuma estratégia de manejo da irrigação até a implantação do Projeto Serviço de Assessoramento ao Irrigante - SAI (SANTOS NETO et al., 2011; LIRA et al., 2011; LIMA et al., 2012). Tal fato conduzia os irrigantes a utilizar esta técnica com total empirismo e sem levar em consideração as peculiaridades locais.

$\mathrm{O}$ agricultor irrigante para realizar uma boa aplicação de água às culturas precisa ter conhecimento das seguintes informações: quando (tempo), quanto (lâmina) e como (método-sistema) irrigar (LIMA et al., 2012). Muitos autores também estudaram a implementação dos serviços de assessoramento e demonstram sua importância para uma irrigação eficiente (ORTEGA et al., 2005; GONZÁLEZDUGO \& MATEOS, 2008; CÓRCOLES et al., 2010; LIMA et. al., 2010; MONTORO et al., 2011; CAR et al. 2012; LORITE et al., 2012; LIMA et. al., 2013b).

No entanto, os SAI's utilizados em todo o mundo somente fornecem informações gerais para os irrigantes, limitando-se ao favorecimento de cálculos ao nível de Evapotranspiração de Referência (ETo) ou de Evapotranspiração da Cultura (ETc). Para realizar o manejo da irrigação do SAI, foi desenvolvido em 2011 um software específico para o sistema. O sistema de computação criado é o Sistema Assessoramento ao Irrigante - S@I. O Sistema S@I possui o banco de dados do DIBAU, a metodologia para as necessidades de água de irrigação computada e o módulo de comunicação. O S@I é um aplicativo exemplar que utiliza a internet para prestar serviços úteis aos agricultores que compõem o DIBAU, oferece oportunidades para a pesquisa, e pode facilmente tornar-se um modelo para muitos outros distritos de irrigação coletiva no Brasil. Além disso, o aplicativo S@ I 
tem o potencial de se transformar em um sistema de apoio à decisão para a gestão de sistemas de irrigação coletivos. O S@I é um sistema disponível na web site (www.sistemasai.com.br) e o processo de envio das informações para os irrigantes tem informações sobre cada usuário e suas propriedades, relacionadas às áreas, número de seções, culturas, sistema de irrigação, água consumida, entre outros. O S@I envia mensagens e e-mails que orientam mais de 300 agricultores do Distrito sobre quando e quanto eles devem irrigar, com o objetivo de maximizar a relação entre o consumo de água e produção de alimentos.

Sousa et al. (2013) avaliaram o funcionamento do sistema, o envio e o recebimento das informações via SMS, no período de agosto de 2012 a abril de 2013, para os produtores cadastrados no SAI. Foram verificadas as possíveis causas da variação na quantidade de mensagens enviadas aos produtores pelo SAI e os erros no sistema S@I. Eles concluíram que ocorreram diferenças no envio devido ao desenvolvimento e das diferentes versões do software, bem como pela sincronia na geração das informações. Os autores ressaltam que o S@I deve ser utilizado como um importante instrumento de manejo da irrigação.

O Sistema S@I é um sistema multidistritos, ou seja, podem ser inseridas novas áreas irrigadas para serem gerenciadas pelo Sistema alternando somente nas ferramentas do software. Desta forma, a gestão da irrigação pode ser feita em grande escala.

Segundo Perry et al. (2009), para entender melhor como as culturas, os ambientes agroclimáticos e as diferentes práticas de manejo podem influenciar na relação entre a produção agrícola e o consumo de água, pode-se definir a produtividade de água para uma cultura (WP) como a razão entre a quantidade produzida e a quantidade de água consumida para obter essa produção. Além disso, a definição da produtividade da água precisa ser específica, indicando qual o produto (rendimento e/ou biomassa) e qual o consumo por transpiração (ou evapotranspiração).

O objetivo desta pesquisa foi avaliar os benefícios do Projeto SAI e das ferramentas do Sistema S@I para a irrigação do DIBAU, em nível de receptividade do irrigante e benefícios para a gestão do Distrito. Desta forma, procurouse avaliar a receptividade de cada irrigante e os benefícios globais que o Sistema pode trazer para todos os produtores através da gestão do uso da água no Distrito. Para mensurar estes benefícios será utilizado o indicador: produtividade de água (WP).

\section{MATERIAL E MÉTODOS}

O Distrito de Irrigação Baixo Acaraú (DIBAU) com sede localizada no Triângulo do Marco distando $220 \mathrm{~km}$ de Fortaleza, na região Norte do estado do Ceará, foi o local de estudo e validação do Sistema S@I. O DIBAU abrange os municípios de Acaraú, Bela Cruz e Marco. O clima da região está caracterizado por Santos Neto et al. (2011) e Lima et al. (2012). Este distrito possui 8.335 ha divididos em 522 lotes, com os agricultores organizados em uma comunidade de irrigantes (Figura 1).

Para realizar o manejo de irrigação no DIBAU foi utilizado o Sistema de Assessoramento ao Irrigante - S@I apresentado por Lima et al. (2013a) que trabalha diretamente na web e pode ser acessado no endereço www.sistemasai.com.br.

Quatro telas do Sistema S@I podem ser visualizadas na Figura 2: (1) visualização global do Distrito; (2) visualização do irrigante escolhido; (3) tela com os dados do irrigante e 4) tela com os dados de Coeficiente da Cultura $(\mathrm{Kc})$.

A partir de dados meteorológicos, 
importados da estação automática do Instituto Nacional de Meteorologia (INMET), é feita a determinação da ETo mediante a metodologia de Penman-Monteith-FAO (ALLEN et al., 1998).

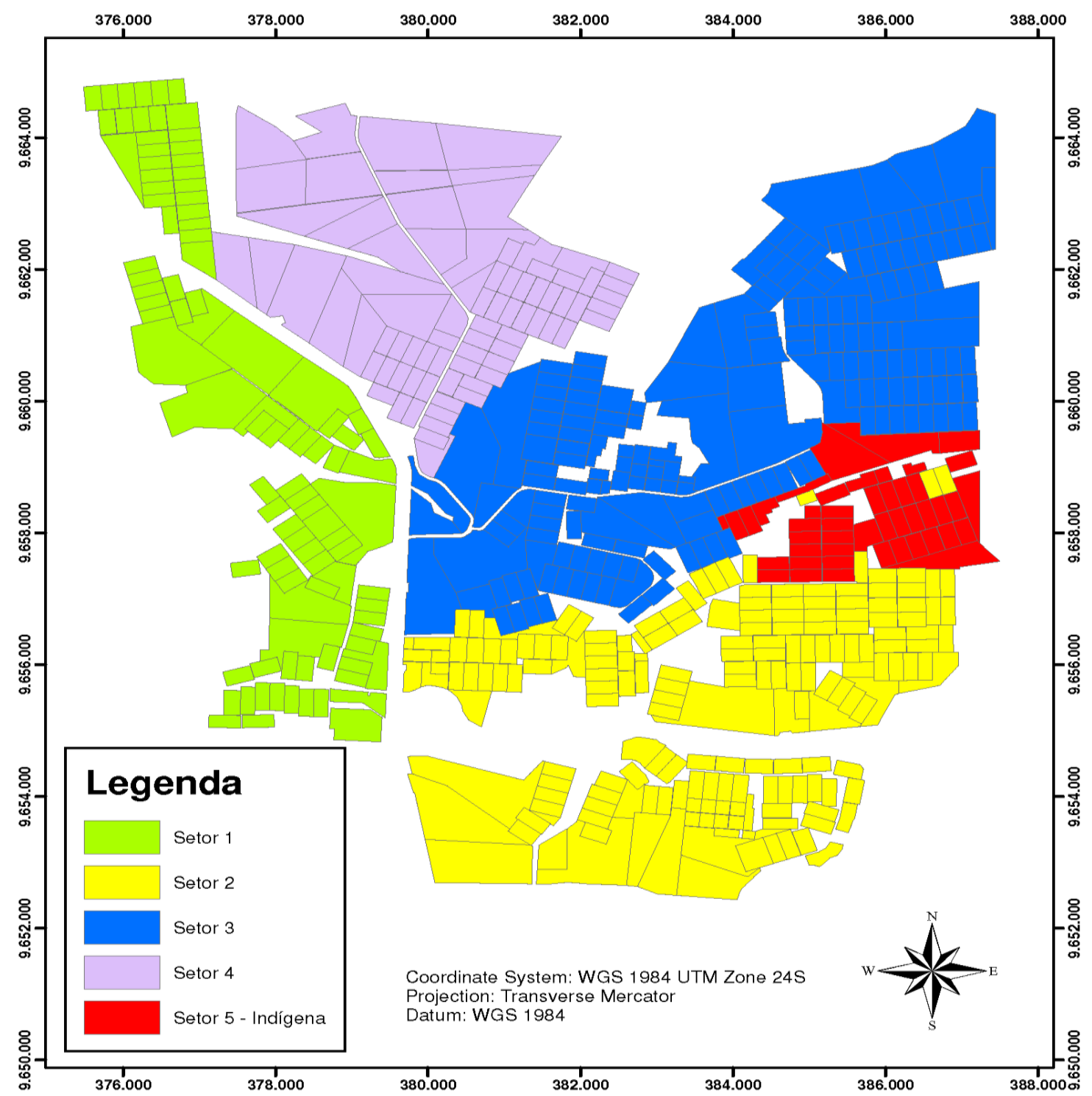

Figura 1. Distrito de Irrigação do Baixo Acaraú dividido por Setores.

O Sistema S@I também armazena e compila dados cadastrais para cada irrigante, tais como, dados do sistema de irrigação, da cultura e informações sobre a propriedade e o agricultor. Além disso, o software pode realizar o balanço hídrico na zona radicular utilizando a precipitação efetiva e a irrigação como parâmetros de entrada de água e a evapotranspiração da cultura (ETc) e a percolação profunda como parâmetros de saída.
De maneira geral, o balanço hídrico pode ser representado pela equação 1 :

$$
\mathrm{WB}=\mathrm{Pe}+\mathrm{I}-\mathrm{ETc}-\mathrm{Pp}
$$

Onde,

WB: balanço hídrico (mm),

P: precipitação efetiva (mm);

I: Lâmina de irrigação (mm);

ETc: evapotranspiração da cultura $(\mathrm{mm})$;

Pp: percolação profunda (mm). 
Lima et al.
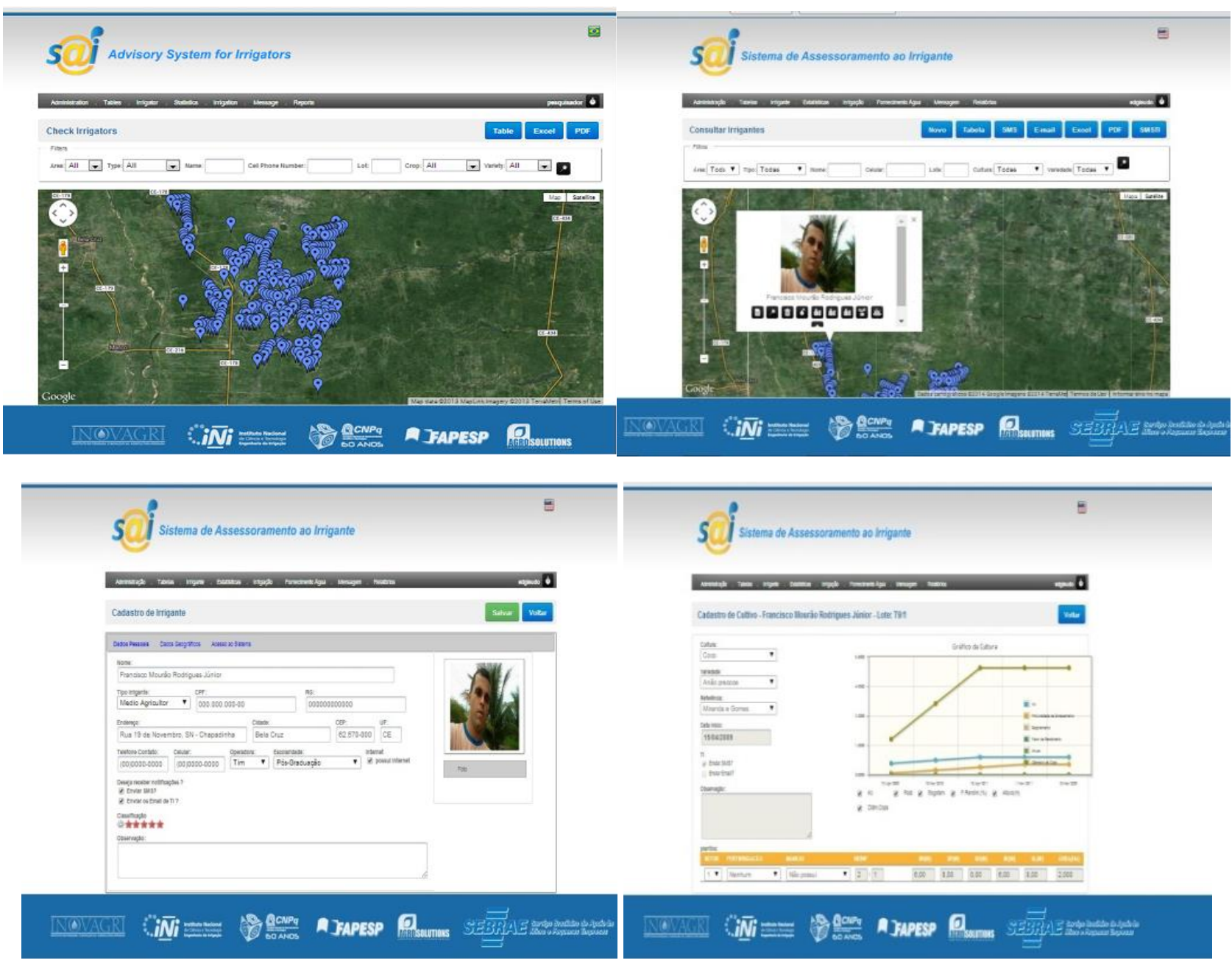

Figura 2. Telas do Sistema S@I

Os coeficientes da cultura $(\mathrm{Kc})$ para $\mathrm{o}$ Distrito foram obtidos por meio de um levantamento do índice por estudos locais ou da região Nordeste. No caso de não haver estudos para determinada cultura, o Kc então foi extraído das tabelas gerais de recomendação do manual FAO-56 (ALLEN et al., 1998). O cálculo das lâminas totais de irrigação a serem aplicadas foi determinado a partir da estimativa da ETc (produto direto entre a evapotranspiração de referência e o coeficiente da cultura). A partir das informações cadastrais de cada propriedade, se obtém as informações da vazão dos emissores utilizados por cada irrigante. Desta forma, diante da compilação de todos os dados de entrada, se calcula o tempo de irrigação recomendado para cada setor da propriedade (LIRA et al., 2011; LIMA et al., 2012).

Diariamente, são gerados os tempos de irrigação para atendimento da necessidade hídrica das culturas. A informação sobre o Tempo de Irrigação (TI) é transferida por mensagens de texto enviadas via SMS e/ou email aos irrigantes do DIBAU cadastrados no Projeto SAI. O irrigante também possui acesso a essas informações e outras mediante uma página web do irrigante desenvolvida no S@I (acessado através de um login e uma senha especialmente criada para o produtor). Um modelo resumido da determinação do TI para este projeto com o modelo similar ao desenvolvido pelo SAI do DIBAU que pode ser visualizada na Figura 3. 


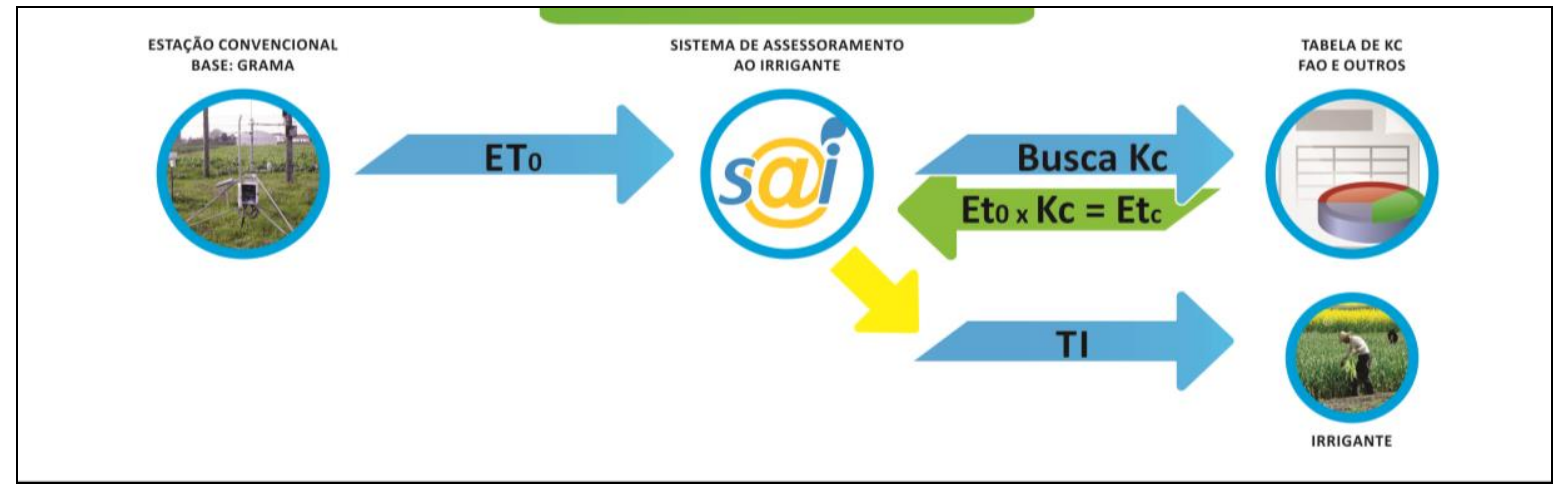

Figura 3. Modelo de determinação da ETc e do Tempo de Irrigação (TI) pelo Sistema S@I

Em se tratando de um solo arenoso como o do DIBAU, neste método de implementação, o balanço de água do S@I não inclui a água armazenada no perfil do solo. Desta forma, evita-se utilizar um sistema complexo que necessitaria contabilizar a umidade do solo, que, em muitas circunstâncias acrescenta pouco benefício como quando as outras preferências agronômicas ou culturais são desconhecidas. $\mathrm{O}$ sistema operacional neste modelo, não informa aos irrigantes quando irrigar, mas fornece uma indicação sobre a quantidade de água a cultura tem utilizado desde a última irrigação. A decisão de quando irrigar cabe ao produtor com base em todas as considerações agronômicas, econômicas e socioculturais. $\mathrm{O}$ produtor pode ter como referência às últimas mensagens enviadas por SMS ou e-mail e aplicar a quantidade adequada e assim fornecer o feedback para o sistema, a fim de atualizar os dados do balanço de água armazenados no servidor.

O Sistema S@I iniciou as atividades de envio em agosto de 2012. Desta forma, para esta pesquisa, foi realizada uma entrevista com os irrigantes cadastrados no Projeto SAI que receberam essas mensagens no período de Agosto de 2012 a Dezembro de 2012. Um formulário foi distribuído no perímetro irrigado nos meses de março a abril de 2013 para todos os 366 usuários cadastrados no sistema. A aplicação desse questionário objetivou conhecer a aceitação do Projeto SAI. As perguntas que foram abordadas na pesquisa foram as seguintes: a) Está recebendo, compreendendo e seguindo as informações de irrigação do SAI?; b) Quais benefícios visuais foram identificados com $\mathrm{o}$ Projeto SAI?; c)Você deseja receber/continuar as informações via SMS ou e-mail? Depois de avaliado, com acompanhamento in loco, o manejo da irrigação no período, bem como analisados os dados dos anos anteriores e definida a eficiência da irrigação e os demais indicadores de uso da água, devem ser introduzidas as avaliações sobre indicadores econômicos e sociais. Para iniciar essa avaliação, definiu-se a evolução dos indicadores econômicos de produtividade de água, pelos dados de volume de água distribuídos pelo DIBAU e os dados indicados pelo Sistema S@I que deveriam ser seguidos pelo Distrito apresentados na equação 2 e equação 3 .

$\mathrm{Na}$ avaliação da produtividade de água realmente utilizada pelo DIBAU, foi definida a equação (2).

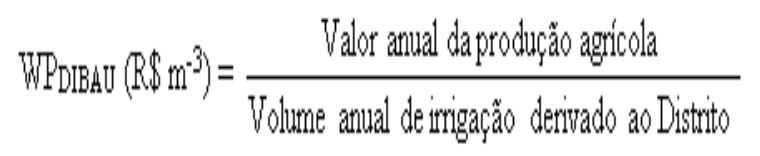

Sendo o Valor anual da produção agrícola obtido através das informações de valor de produção repassadas pelos irrigantes ao Distrito e o volume anual da irrigação derivado ao 
DIBAU foi obtido através dos dados de medição coletados pelo próprio distrito e fornecidos ao SAI para a pesquisa.

$\mathrm{Na}$ avaliação da produtividade de água indicado pelo S@I, caso o Distrito utilizasse na época, foi definida a equação (3).

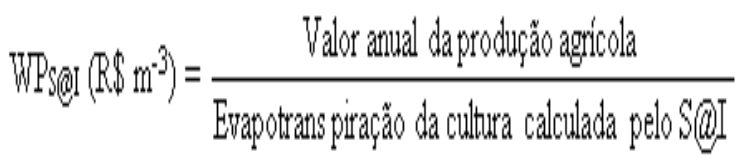

Como a evapotranspiração da cultura é dada em mm, esta foi multiplicada pela área para obter o volume em metros cúbicos.

\section{RESULTADOS E DISCUSSÃO}

Há duas barreiras críticas específicas relacionadas com a água: uma está relacionada com as grandes perdas de água na irrigação e a outra se refere à insuficiente informação dos agricultores sobre o seu manejo adequado. A irrigação é a atividade de maior consumo de água com potencial de crescimento significativo e uso nem sempre racional dos recursos hídricos. Portanto, é necessário encontrar formas de aumentar a eficiência do uso da água nos distritos de irrigação. O Sistema S@I apresenta uma proposta que é o pedágio inteligente para superar este problema, como a realização de cálculos que são enviados aos agricultores e que os ensinam a quantidade de água a ser aplicada. A outra barreira crítica específica importante está relacionada com o uso da água destinada à segurança alimentar devido à falta informação e a dificuldade dos agricultores em utilizar inovações tecnológicas.

Durante o período de envio inicial das mensagens, o DIBAU se dividia entre as quatro principais culturas produzidas no Distrito: 1.495,08ha de Coco, 618,40ha de Banana, 504,00ha de Laranja e 230,45ha de Goiaba.

De acordo com Sousa et al. (2013), as mensagens com os tempos de irrigação informados aos irrigantes, efetivamente, iniciaram-se a partir do mês de agosto de 2012 . Segundo o autor, o número de irrigantes que compreende a informação é em torno de $97 \%$ e alguns aspectos contribuíram para a obtenção deste expressivo número, como por exemplo, a escrita dos caracteres de fácil compreensão e o recebimento da informação via e-mail, porém, somente $35,78 \%$ seguem as recomendações. Mesmo assim, esse é um valor significativo por se tratar de um projeto pioneiro na região e ter embasamento dos cálculos somente levando em consideração os dados climáticos.

$\mathrm{Na}$ Tabela 1, dentro do universo dos irrigantes apresentados por Sousa et al. (2013), o resultado da entrevista deste trabalho mostrou que em relação aos que utilizam os tempos de irrigação recebidos, aproximadamente $31 \%$ os seguem diariamente. Outros $41,02 \%$ utilizam como uma referência para realizar a irrigação, podendo irrigar mais ou menos, de acordo com a conveniência dele.

Tabela 1. Levantamento da forma de utilização dos SMS recebidos

FORMA DE UTILIZACAO DO TEMPO DE $\%$
IRRIGAÇÃO RECEBIDO

Seguem como referência 41,02

Seguem diariamente 30,77

Seguem sempre que possível 28,21

Dentre os benefícios citados pelos irrigantes que seguem as recomendações dos tempos de irrigação enviados pelo Projeto SAI, observou-se uma melhora visual de $25,64 \%$ das culturas irrigadas (Tabela 2). No entanto, a maioria dos entrevistados não observou nenhuma alteração. Como a cultura principal produzida no distrito é o Coco (Cocos nucifera L.), conforme apresentado anteriormente, essa identificação 

GESTÃO DA ÁGUA EM ÁREAS IRRIGADAS: BENEFÍCIOS AOS IRRIGANTES E AO DISTRITO

torna-se difícil em tão pouco tempo de atividades do Projeto SAI.

Tabela 2. Levantamento dos benefícios na cultura

\section{BENEFICIOS IDENTIFICADOS NA \% CULTURA}

Melhora no aspecto visual $\quad 25,64$

Não percebeu nenhuma alteração

74,36

Em relação ao último quesito da entrevista, a pesquisa identificou que os irrigantes desejam continuar recebendo a informação, porém aproximadamente $20 \%$ dos irrigantes estão optando por receber a informações do tempo de irrigação via e-mail. A amostragem demonstrou resultado similar ao relatado por Car et al. (2012). Aquele autor, ao final da temporada, aplicou uma entrevista com os usuários e observou que $80 \%$ dos irrigantes pretendem utilizar a mensagem, mas não integralmente e sim como um apoio à tomada de decisão, já que outras características devem ser levadas em consideração, principalmente o aspecto visual das plantas.

Um grande número de usuários tem acesso aos principais dados climáticos diários fornecidos por qualquer uma das estações micrometeorológicas do SAI, e com isso se torna possível executar médias por períodos ou consultar dados históricos. Este aspecto é uma ferramenta importante de ajuda, não só para estimar as necessidades hídricas das culturas, mas também para a tomada de decisões sobre as datas de plantio e a seleção de variedades. O número de agricultores acessando a página da Web vem aumentando gradualmente, devido a uma crescente incorporação de novas tecnologias. Aplicando o cálculo correto para as necessidades hídricas, será possível, em uma escala completa de região, permitir que qualquer agricultor maneje corretamente a irrigação. Desta forma, haverá um Serviço de Assessoramento ao Irrigante implantado disseminado para todos os irrigantes do Distrito de Irrigação Baixo Acaraú (DIBAU), a aplicação eficiente e uniformidade da água ao nível de lote, elevados níveis de produtividade das culturas exploradas, elevação dos níveis de capacitação dos produtores como resultado de cursos e das práticas de manejo racional da água oriundas da implantação do SAI e, finalmente, realizar pesquisas para a obtenção de conceitos sobre qualidade do manejo da irrigação para o agronegócio brasileiro.

Depois de analisar os dados fornecidos de derivação de água ao Distrito, foi possível calcular alguns indicadores de uso da água, especialmente o WP. Neste caso, é possível medir o WP DIBAU (Derivado ao Distrito) e WP S@I (calculado pelo sistema). Para uma análise mais detalhada o projeto foi dividido em três fases:

$\mathrm{Na}$ primeira fase, a análise dos dados foi concentrada nos primeiros 5 meses de implementação do S@I (Ago-Dec/2012). Observou-se que no Distrito (DIBAU) o consumo de água foi $22.620 .408 \mathrm{~m}^{3}$ (efetivamente para irrigação), como registrado nos hidrômetros instalados na área de muitos agricultores. Este valor foi maior do que a recomendada pelo sistema S@I, que forneceu uma exigência hídrica da cultura, de $13.404 .788 \mathrm{~m}^{3}$ (59,26\% do total medido de água aplicada). Se os agricultores do DIBAU tivessem utilizado a recomendação S@I, o Distrito teria uma economia de $9.215 .620 \mathrm{~m}^{3}$ de água.

$\mathrm{Na}$ segunda fase (Ago/2012-Jul/2013), quando se mediu a aplicação de água do Distrito de Irrigação se observou que a mesma foi novamente superior $\left(43.220 .476 \mathrm{~m}^{3}\right)$ quando comparada à recomendação do sistema S@I $\left(31.762 .753 \mathrm{~m}^{3}\right)$, que se baseia no cálculo das 
necessidades hídricas das culturas. Essa superioridade foi equivalente a $73.5 \%$ do volume total recomendado pelo programa, sendo assim, se os irrigantes tivessem seguido a recomendação do S@I, o Distrito teria obtido uma economia de 11.457.724 $\mathrm{m}^{3}$ de água durante esse período.

A aplicação de água na terceira fase desta análise foi $45.513 .148 \mathrm{~m}^{3}$, o que se mostrou mais uma vez superior ao valor recomendado pelo S@I para a necessidade de água das culturas $\left(38.157 .401 \mathrm{~m}^{3}\right)$, equivalente a $83,8 \%$ da água aplicada medida, representando uma possível economia de $7.355 .748 \mathrm{~m}^{3}$ de água.

De acordo com as análises da evolução da produtividade de água (WP) demonstradas na Figura 4, pode-se observar uma divergência desse índice ao longo das três fases do projeto. A diferença entre a $1^{\text {a }}$ Fase e a $2^{\text {a }}$ Fase justifica-se pela abrangência somente do período em que se praticava a irrigação durante a $1^{\mathrm{a}}$ Fase. Já na $2^{\mathrm{a}}$
Fase, foi contabilizado também o período em que não se irrigava, e como consequência se eleva a produtividade de água visto que seu consumo foi evidentemente menor. Entre a $2^{\mathrm{a}}$ Fase e a $3^{\text {a }}$ Fase, foi observado um aumento da produtividade da água nos dois indicadores (DIBAU e S@I), tal elevação do índice pode ser justificada devida à implantação de novas culturas e a necessidade de se economizar água na Região, visto que o ano de 2013 foi um ano de uma baixa recarga hídrica em todo Estado do Ceará.

De qualquer forma a WP do DIBAU variou de $0,68 \mathrm{R} \$ \cdot \mathrm{m}^{-3}$ a $1,15 \mathrm{R} \$ . \mathrm{m}^{-3}$ e WP do $\mathrm{S} @ \mathrm{I}$ variam de $1,19 \mathrm{R} \$ . \mathrm{m}^{-3}$ a $1,38 \mathrm{R} \$ . \mathrm{m}^{-3} . \mathrm{Em}$ todas as situações a WP S@I foi mais alta do que a WP DIBAU demonstrando que terá maior valor de produção por metro cúbico de água se os produtores irrigarem seguindo a recomendação do Sistema S@I.

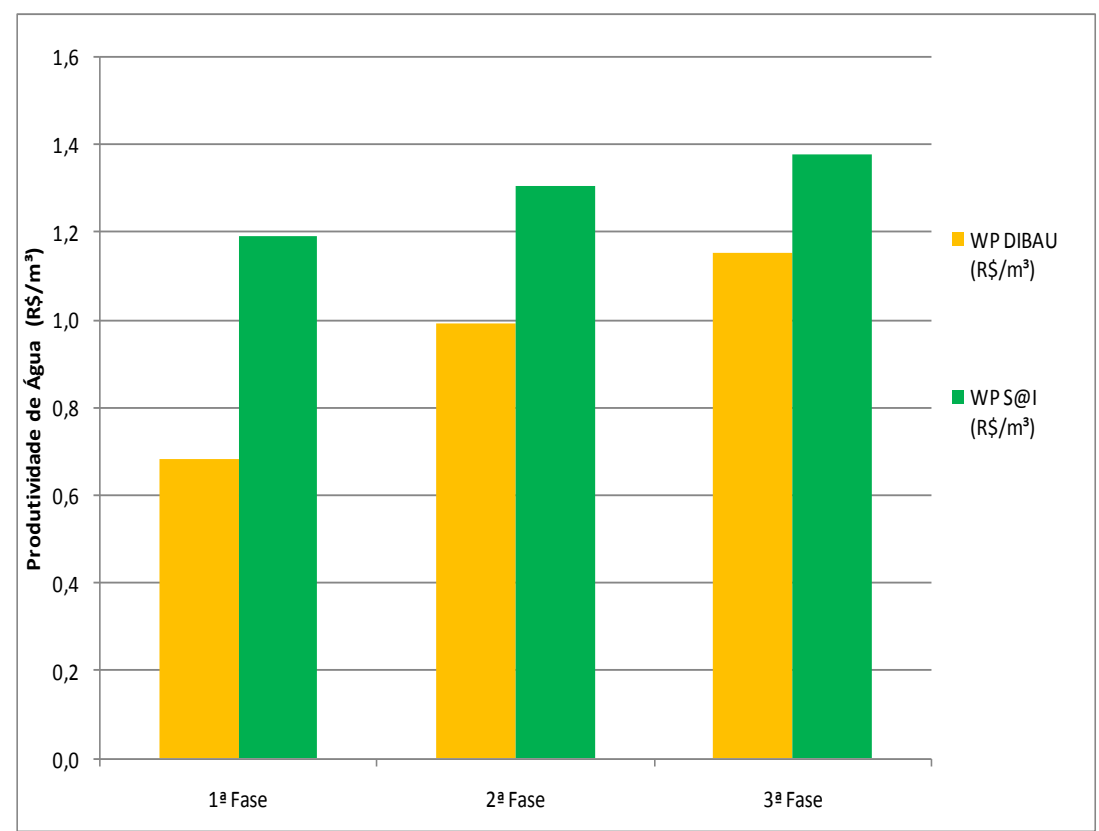

Figura 4. Resultado da Produtividade de Água no DIBAU obtida pelo S@I

Desde o início dos trabalhos do SAI no DIBAU já se constatava que o uso da água era deficiente naquele Distrito. Com a implantação do SAI, os agricultores receberam orientações dos técnicos e diversos setores tiveram sua uniformidade de emissão de água melhorada. Santos Neto et al. (2011), avaliando a eficiência do uso da água nos sistemas de irrigação dos 
colonos do DIBAU encontrou uma evolução em alguns lotes de $9 \%$ para $90 \%$. Lima et al. (2013b) identificaram uma evolução na qualidade da irrigação no DIBAU após as inserções das atividades do SAI. Mesmo assim, observa-se que ainda é necessária uma gestão do Distrito em paralelo com a gestão individual dos irrigantes. A análise dos dados do sistema S@I têm comprovado que este sistema pode ser utilizado como uma referência para demonstrar que a eficiência do uso da água no Distrito de Irrigação pode ser melhorada.

\section{CONCLUSÃO}

1. O Projeto SAI com a utilização do Sistema S@I auxilia na gestão eficiente do uso da água, levando em consideração a identificação do consumo demandado pelas culturas implantadas.

2. O Distrito está perdendo água, pois a WP calculada pelo S@I comprovou que o DIBAU deriva um volume maior do que o necessário para as culturas. Esta informação deve servir de referência ao Distrito que com as devidas adequações pode economizar água e energia, gerando um benefício aos irrigantes e à área coletiva de irrigação.

3. O sistema S@I se mostrou uma excelente ferramenta de transferência de tecnologia, mesmo com a falta de dados para uma melhor conclusão em relação aos lotes de cada agricultor.

\section{AGRADECIMENTOS}

Os autores agradecem ao Instituto Nacional de Ciência e Tecnologia em Engenharia da Irrigação (INCT-EI), ao CNPq, à FAPESP e ao Distrito de Irrigação do Baixo Acaraú (DIBAU).

\section{REFERÊNCIAS BIBLIOGRÁFICAS}

ALLEN, R.G.; PEREIRA, L.S.; RAES, D.; SMITH, M. Crop evapotranspiration: guidelines for computing crop water requirements. Irrigation and Drainage. Paper 56. FAO, Rome, Italy, 1998.

BARROS, R.; ISIDORO, D.; ARAGÜÉS, R. Long-term water balances in La Violada irrigation district (Spain): I. Sequential assessment and minimization of closing errors. Agricultural Water Management, n. 98, p. 1569-1576, 2011.

CAR, N. J.; CHRISTEN, E. W.; HORNBUCKLE, J. W.; MOORE, G. A. Using a mobile phone Short Messaging Service (SMS) for irrigation scheduling in Australia - Farmers' participation and utility evaluation. Computers and Electronics in Agriculture, n. 84, p. 132143, 2012.

CÓRCOLES, J. I.; DE JUAN, J. A.; ORTEGA, J. F.; TARJUELO, J. M.; MORENO, M. A. Management evaluation of Water Users Associations using benchmarking techniques. Agricultural Water Management, n. 98, p. 111, 2010.

GONZÁLEZ-DUGO, M. P.; MATEOS, L. Spectral vegetation indices for benchmarking water productivity of irrigated cotton and sugarbeet crops. Agricultural Water Management, n. 95, p. 48-58, 2008.

ISIDORO, D.; QUÍLEZ, D.; ARAGÜÉS, R. Water balance and irrigation performance analysis: La Violada irrigation district (Spain) as a case study. Agricultural Water Management, n. 64, p. 123-142, 2004.

LIMA, S. C. R. V.; FRIZZONE, J. A.; MATEOS, L.; FERNANDEZ. M. S. Desempenho da Irrigação em um Projeto Hidroagrícola no Sul da Espanha: metodologia para análise da eficiência de uso da água. 
Revista Brasileira de Agricultura Irrigada, v. 4, p. 59-77, 2010.

LIMA, S. C. R. V.; FRIZZONE, J. A.; SOUSA, A. E. C.; BELTRÃO JÚNIOR, J. A.; FERREIRA, R. P.; GARCIA, D. R. Aplicação da tecnologia da informação e a adoção pelo agricultor: a avaliação inicial do envio de mensagens pelo serviço de assessoramento ao irrigante. Revista Brasileira de Agricultura Irrigada, v. 6, p. 314-328, 2012.

LIMA， S. C. R. V.; FRIZZONE， J. A.; SNYDER, R. L.; MATEOS, L. Evolution of irrigation quality after the assistance for farmers: the experience of the advisory service for irrigators - SAI in "Baixo Acaraú" Irrigation District. In: 1st CIGR Inter-Regional Conference On Land and Water Challenges, 2013, Bari. - 1st CIGR Inter-Regional Conference On Land and Water Challenges. Bari: IAMB, 2013a. Proceedings

LIMA, S. C. R. V.; FRIZZONE, J. A.; SANTOS NETO, A. M.; BELTRÃO JÚNIOR, J. A.; RODRIGUES JÚNIOR, F. M.; NUNES FILHO, J. L. N. Evolução da qualidade da irrigação após as atuações do serviço de assessoramento ao irrigante. Revista Brasileira de Agricultura Irrigada, v. 7, p. 91-101, 2013 b.

LIRA, J. V.; MOURÃO JÚNIOR, F.; SOUSA, A. E. C.; LIMA, S. C. R. V.; FRIZZONE, J. A.; VIANA, J. M. Auditoria de Desempenho de Sistemas de Irrigação I: A experiência inicial do Serviço de Assessoramento ao Irrigante - SAI, no Distrito de Irrigação do Baixo Acaraú. Revista Brasileira de Ágricultura Irrigada, v. 5, p. 265-271, 2011.

LORITE, I. J.; GARCÍA-VILA, M.; CARMONA, M. A.; SANTOS, C.; SORIANO, M. A. Assessment of the Irrigation Advisory Services' Recommendations and Farmers' Irrigation Management: A Case Study in Southern Spain. Water Resources Management, v. 26, p. 2397-2419, 2012.
LOZANO, D.; MATEOS, L. Usefulness and limitations of decision support systems for improving irrigation scheme management. Agricultural Water Management, v. 95, p. 409-418, 2008.

MONTORO, A., LÓPEZ-FUSTER, P., FERERES, E. Improving on-farm water management through an irrigation scheduling service. Irrigation Science, v. 29, p. 311-319, 2011.

ORTEGA, J. F.; DE JUAN, J. A.; TARJUELO, J. M. Improving water management: The irrigation advisory service of Castilla-La Mancha (Spain). Agricultural Water Management, v. 77, p. 37-58, 2005.

PERRY, C.; STEDUTO, P.; ALLEN, R. G.; BURT, C. M. Increasing productivity in irrigated agriculture: Agronomic constraints and hydrological realities. Agricultural Water Management, v. 96, p. 1517-1524, 2009.

RODRÍGUEZ-DÍAZ， J. A.; CAMACHOPOYATO, E.; LÓPEZ-LUQUE, R.; PÉREZURRESTARAZU, L. Benchmarking and multivariate data analysis techniques for improving the efficiency of irrigation districts: An application in Spain. Agricultural Systems, v. 96, p. 250-259, 2008.

SANTOS NETO, A. M.; BRAGA, A. C. C.; SILVA, M. M. M.; LIMA, S. C. R. V.; FRIZZONE, J. A.; GOMES FILHO, R. R. Auditoria de Desempenho de Sistemas de Irrigação II: Avaliação e Correção da Uniformidade de Emissão de Água no Distrito de Irrigação do Baixo Acaraú, CE. Revista Brasileira de Agricultura Irrigada, v. 5, p. 272-279, 2011.

SNYDER, R.L. California Irrigation Management Information System. American Potato Journal, 61: 229-234, 1984.

SOUSA, E. C.; SANTOS NETO, A. M.; LIMA, S. C. R. V.; FRIZZONE, J. A.; NASCIMENTO, 
DESEMPENHO DA UTILIZAČ̃̃O DO SISTEMA DE ASSESSORAMENTO AO IRRIGANTE - S@I PARA A GESTÃO DA ÁGUA EM ÁREAS IRRIGADAS: BENEFÍCIOS AOS IRRIGANTES E AO DISTRITO

A. K. S.; BELTRÃO JÚNIOR, J. A. Utilização da tecnologia da informação para o envio de mensagens via SMS ao irrigante: a experiência inicial do projeto SAI. Revista Brasileira de Agricultura Irrigada, v. 7, p. $224-234$, 2013. 